\title{
Myopic Simple Hemorrhage Presenting as Radial Hemorrhage in Henle's Fiber Layer
}

\author{
Marco Mazzola a, b Laura Premoli ${ }^{a}$ Cristian Metrangolo ${ }^{a}$ \\ Jennifer Cattaneo ${ }^{c} \quad$ Elias Premi $^{a} \quad$ Simone Donati $^{a} \quad$ Claudio Azzolini $^{a}$ \\ aOphthalmology Clinic, Department of Medicine and Surgery, University of Insubria, \\ Varese-Como, Italy; ${ }^{b}$ Ophthalmology Department, IRCCS Istituto Auxologico Italiano, Milan, \\ Italy; ' Ophthalmology Clinic, Department of Neuroscience, ASST Ovest Milanese, Legnano, \\ Italy
}

\section{Keywords}

Eye disease · Imaging · Macula/retina/vitreous · Maculopathy · Myopia · Ophthalmoscopy Simple hemorrhage $\cdot$ Radial hemorrhage $\cdot$ Henle fiber layer hemorrhage $\cdot$ Myopic hemorrhage $\cdot$ Retina

\begin{abstract}
This case report describes a simple hemorrhage $(\mathrm{SH})$ presenting as radial hemorrhage in Henle's fiber layer (HFL) in a patient with high myopia. A 26-year-old girl with high myopia was referred to our center for sudden onset of decreased vision and a central scotoma in the right eye (OD). Best corrected visual acuity (BCVA) was 20/100 OD. Fundus examination showed a stellate intraretinal hemorrhage in the fovea of the OD. The hemorrhage was organized in a peculiar petaloid pattern with feathery distal edges, suggesting localization within the radially oriented HFL. The presence of both choroidal neovascularization and microvascular abnormalities consistent with macular telangiectasia type 2 (MacTel 2) were excluded. Based on these findings, a diagnosis of myopic SH was made. At 4-month follow-up BCVA OD spontaneously improved to 20/40, without any treatment been ever administered to the patient. Spectral-domain optical coherence tomography OD showed reabsorption of the hemorrhage and almost complete restoration of the foveal architecture. The intraretinal location and spread of the hemorrhage into the HFL in our patient are an unusual presentation of $\mathrm{SH}$, which vividly highlights the anatomy of the fovea. Since fibers in HFL are quite delicate and loosely arranged, this layer is very susceptible to deposition of transudates, exudates, hemorrhage, and other products. Radial hemorrhage in HFL has been originally reported in 4 patients as complication of MacTel 2. It has been previously postulated that it may represent a characteristic finding in MacTel 2 that may develop as a result of microvascular abnormalities of the
\end{abstract}


deep retinal capillary plexus. On the contrary, our data suggest that radial hemorrhage in the HFL does not represent a characteristic finding of MacTel 2, but must rather be considered a non-specific sign with multiple possible etiologies.

(C) 2021 The Author(s).

Published by S. Karger AG, Basel

\section{Introduction}

Within the outer plexiform layer (OPL), the photoreceptor axons are known as Henle's fiber layer (HFL). Radial hemorrhage in HFL has been originally reported in 4 patients as complication of macular telangiectasia type 2 (MacTel 2) [1, 2]. It has been previously postulated that it may represent a characteristic finding in MacTel 2 that may develop as a result of microvascular abnormalities of the deep retinal capillary plexus in the absence of choroidal neovascularization (CNV) [2].

Two types of subretinal hemorrhage, with and without CNV, have been reported in high myopia. Simple hemorrhage (SH) is a term used for macular bleeding without a CNV that occurs in highly myopic eyes and is most likely caused by the mechanical rupture of Bruch membrane and choriocapillaris complex. Importantly, simple macular bleeding is often misdiagnosed as a myopic CNV and consequently mistreated with intravitreal injections of antiVEGF [3].

\section{Case Presentation}

A 26-year-old girl with high myopia was referred to the Retina Service, Ophthalmology Clinic, University of Insubria - ASST Sette Laghi, Varese, Italy for sudden onset of decreased vision and a central scotoma in the right eye (OD). She denied recent trauma or Valsalva maneuver. She was in good health and was not affected by any systemic disease except for panic disorder, for which she was taking oral alprazolam and escitalopram. She underwent a routine blood test a couple of months before she was referred to our unit, and all complete blood count parameter were within normal range.

Best corrected visual acuity (BCVA) was 20/100 OD and 20/20 in the left eye (OS). Spheric equivalent refractive error was -10.0 diopters in both eyes.

Fundus examination (Fig. 1a) showed a stellate intraretinal hemorrhage in the fovea of the OD. The hemorrhage was organized in a peculiar petaloid pattern with feathery distal edges, suggesting localization within the radially oriented HFL. OS was unaffected.

Spectral-domain optical coherence tomography (SD-OCT) of the OD showed an elevated hyper reflective lesion in the outer retina, with areas of shadowing of the underlying retinal pigment epithelium (RPE) (Fig. 1b). Intraretinal fluid within the OPL was also noted adjacent to the lesion (Fig. 1c).

OCT Angiography OD was performed 4 days later, and excluded the presence of both CNV and microvascular abnormalities consistent with MacTel 2 (Fig. 1d). Based on these findings, a diagnosis of myopic SH was made. En face OCT of the OD illustrated radial hyperreflectivity at the level of the HFL (Fig. 1e). Cross-sectional OCT of the OD displayed intraretinal hemorrhage radiating in the HFL (Fig. 1f).

At 1-month follow-up BCVA OD dropped to 20/200. SD-OCT OD (Fig. 2a) showed partial reabsorption of the intraretinal hemorrhage, with normalization of retinal profile and restoration of the foveal pit. Hyper reflective material was still present in the subfoveal region, with disruption of the outer retinal layers.

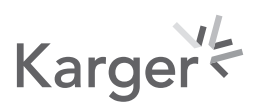




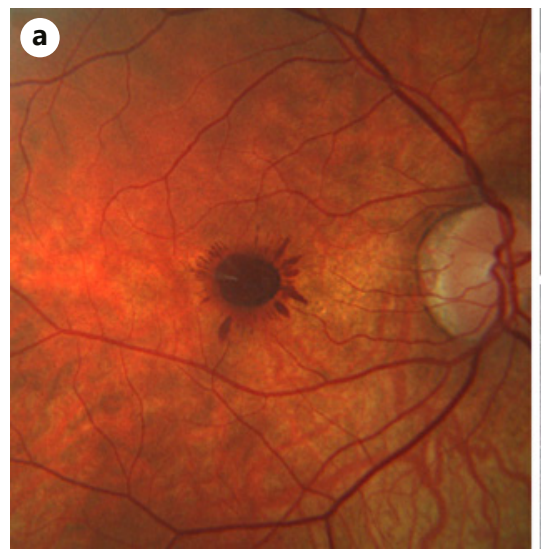

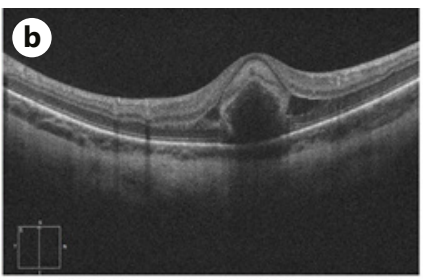

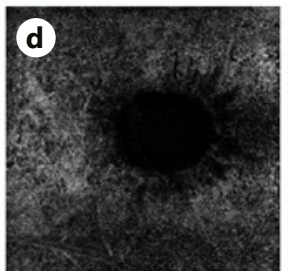

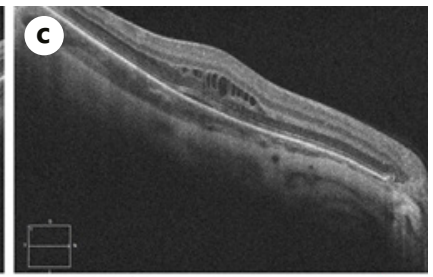
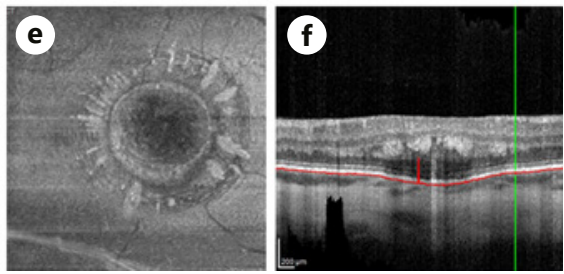

Fig. 1. Baseline multimodal imaging. a Color fundus photography displays macular hemorrhage in a characteristic radial pattern. b, c Spectral-domain optical coherence tomography shows an elevated hyper reflective lesion in the outer retina, with areas of shadowing of the underlying retinal pigment epithelium and accumulation of fluid within the outer plexiform layer adjacent to the lesion. d Optical coherence tomography angiography shows no sign of choroidal neovascularization nor microvascular abnormalities consistent with macular telangiectasia type 2. e En face optical coherence tomography reveals radial hyperreflectivity at the level of the outer plexiform layer. $\mathbf{f}$ Cross-sectional optical coherence tomography displays intraretinal hyper reflective material radiating in the outer plexiform layer.
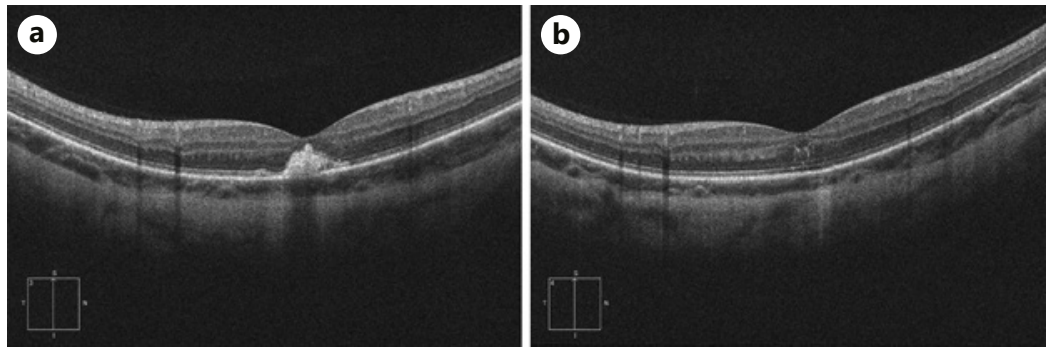

Fig. 2. One-month and 4-month follow-up. a One-month follow-up. Spectral-domain optical coherence tomography shows partial reabsorption of the intraretinal hemorrhage. Hyper reflective material is still present in the subfoveal region, with disruption of the outer retinal layers. b Four-month follow-up. Spectral-domain optical coherence tomography shows intraretinal hyperreflective signs perpendicular to the retinal pigmented epithelium in the foveal region, with subtle disruption of the inner segment/ outer segment layer.

At 4-month follow-up BCVA OD spontaneously improved to 20/40, without any treatment been ever administered to the patient. SD-OCT OD (Fig. 2b) showed intraretinal hyperreflective signs perpendicular to the RPE in the foveal region, with subtle disruption of the inner segment/outer segment layer. HFL was clearly visible inferiorly to the fovea.

\section{Discussion and Conclusion}

The intraretinal location and spread of the hemorrhage into the HFL in our patient are an unusual presentation of $\mathrm{SH}$. The presence of intraretinal fluid is probably due to mechanical stress, but could be misleading and wrongly attributed to the presence of CNV. 
Our data seem to contrast with the original theory postulated by Au et al. [2], that radial hemorrhage in the HFL represent a characteristic finding of MacTel 2, that may develop as a result of microvascular abnormalities of the deep retinal capillary plexus. On the contrary, our findings suggest that radial hemorrhage in the HFL is rather linked with the peculiar anatomy of the fibers in HFL, which are quite delicate and loosely arranged, and therefore are very susceptible to deposition of transudates, exudates, hemorrhage, and other products [4]. Potentially, any kind of hemorrhage which involve HFL could led to the peculiar appearance of a macular radial hemorrhage, which cannot therefore be considered pathognomonic of any particular disease but must rather be considered a nonspecific sign with multiple possible etiologies, as also suggested by Baumal et al. in their recent case series [5].

As for its natural history, the typical $\mathrm{SH}$, which occurs without development of $\mathrm{CNV}$, has a relatively favorable outcome unless hemorrhage recurs, atrophic scars develop, or retinochoroidal degeneration progresses [6].

Multimodal imaging is of fundamental importance for the correct differential diagnosis and treatment of macular hemorrhages in highly myopic eyes.

\section{Statement of Ethics}

This research complied with the guidelines for human studies and was conducted ethically in accordance with the World Medical Association Declaration of Helsinki. Written informed consent for publication of this case report and any accompanying images was obtained from the patient.

\section{Conflict of Interest Statement}

The authors have no conflicts of interest to declare.

\section{Funding Sources}

There was no funding source for this study.

\section{Author Contributions}

M.M.: patient management, conception of the paper, data analysis and interpretation, manuscript writing, literature search. L.P. and C.M.: patient management, data analysis and interpretation, literature search. J.C.: patient management, data analysis and interpretation. E.P.: data analysis and interpretation, literature search. C.A. and S.D.: manuscript drafting, literature search, manuscript editing and revision. All authors read and approved the final manuscript.

\section{References}

1 Rahimy E, Vander J. I see a seashell in my right eye. JAMA Ophthalmol. 2014 Dec;132(12):1413.

2 Au A, Hou K, Baumal CR, Sarraf D. Radial hemorrhage in henle layer in macular telangiectasia type 2. JAMA Ophthalmol. 2018 Oct 1;136(10):1182-5.

3 Asai T, Ikuno Y, Nishida K. Macular microstructures and prognostic factors in myopic subretinal hemorrhages. Invest Ophthalmol Vis Sci. 2014 Jan 9;55(1):226-32.

\section{Karger'}


4 Nauman GOH, Apple DJ. Microscopic anatomy of the eye. Pathology of the eye. New York, NY: Springer-Verlag; 1985. p. 43.

5 Baumal CR, Sarraf D, Bryant T, Gui W, Muakkassa N, Pichi F, et al. Henle fibre layer haemorrhage: clinical features and pathogenesis. Br J Ophthalmol. 2021;105(3):374-80.

6 Goto S, Sayanagi K, Ikuno Y, Jo Y, Gomi F, Nishida K. Comparison of visual prognoses between natural course of simple hemorrhage and choroidal neovascularization treated with intravitreal bevacizumab in highly myopic eyes: a 1-year follow-up. Retina. 2015 Mar;35(3):429-34. 